Original

\title{
Sarcomas paratesticulares en el paciente adulto. Manejo y evolución de la enfermedad
}

\author{
Juan C. Astigueta Pérez*, Milagros Abad Licham**, Mariela Pow-Sang Godoy***, \\ Víctor Destefano Urrutia***, Juvenal Sánchez Lihon** \\ *Departamento de Cirugía Urológica, Instituto Regional de Enfermedades Neoplásicas, Trujillo, Perú. \\ Departamentos de **Patología y ${ }^{* * *}$ Urología Oncológica, Instituto Nacional de Enfermedades Neoplásicas. Lima, Perú.
}

\begin{abstract}
Resumen
Objetivo: Identificar y analizar las características clínico-patológicas, terapéuticas y la evolución de la enfermedad en pacientes adultos con diagnostico de sarcoma paratesticular.

Material y Métodos: Se revisaron retrospectivamente las historias clínicas de los pacientes con diagnóstico de sarcoma, de localización paratesticular del departamento de Urología del Instituto Nacional de Enfermedades Neoplásicas desde 1952 hasta el 2003. Se recopiló datos de filiación, antecedentes, sintomatología, métodos diagnósticos, estudios anatomopatologicos, tratamientos y estado de la enfermedad. Se analizó estadísticamente y evaluó comparativamente con la información obtenida en la revisión de la literatura.

Resultados: Se encontraron dieciséis casos de sarcoma paratesticular en pacientes mayores de 16 años, 9 rabdomiosarcomas, 3 leiomiosarcomas, 3 liposarcomas y 1 sarcoma no clasificable. Hubo dos picos de presentación, uno entre 16 y 20 años y otro en mayores de 60 años. Seis tenían enfermedad metastásica al diagnóstico. La sobrevida en promedio fue de 32,18 meses con un rango entre 1 y 142 meses, sólo dos pacientes se encuentran vivos, un caso de rabdomiosarcoma sin evidencia de enfermedad y otro de leiomiosarcoma con recurrencia a nivel del canal inguinal (zona de cirugía).

Conclusiones: Los sarcomas paratesticulares son una neoplasia maligna rara de pobre pronóstico. El comportamiento biológico del tumor y la historia de enfermedad son variables de acuerdo al subtipo histológico, pero en general la evolución es tórpida con desenlace fatal.
\end{abstract}

Palabras clave: Sarcoma. Paratesticular. Adulto.

\section{Adult paratesticular sarcomas. Management and evolution of the disease}

\section{Abstract}

Objective: Identify and analyze the clinic pathologic characteristics, therapeutic, and the evolution of the disease in adult patients with a diagnosis of paratesticular sarcoma.

Material and Methods: We reviewed retrospectively the medical records of patients diagnosed with sarcoma paratesticular location of the department of urology at the National Institute of Neoplastic Diseases from 1952 to 2003. Data was collected affiliation, background, symptoms, diagnostic methods, pathological diagnosis, treatment and status of the disease. We analyzed statistically evaluated and compared with information obtained in the literature.

Results: Sixteen paratesticular sarcomas cases were found in patients greater than 16 years old; 9 rhabdomyosarcomas, 3 leiomyosarcomas, 3 liposarcomas, and 1 nonclassifiable sarcoma. There were two thresholds of presentation, one among 16 to 20 years and another one in greater than 60 years. Six had metastatic disease at diagnosis. The average survival was 32.18 months with a range among 1 and 142, only two patients are alive, a case of rhabdomyosarcoma without disease evidence and another one of leiomyosarcoma with inguinal recurrence.

Conclusions: Paratesticular sarcoma is a rare malignant neoplasia of poor prognostic. with variable history of disease in relation a histology subtype, but in general of bad evolution with fatal ending. The biological behavior of the tumor and history of the disease vary according to histological subtype, but in general the trend is poor with fatal outcome.

Keywords: Sarcoma. Paratesticular. Adult.

$\mathrm{L}^{\mathrm{s}}$ os tumores malignos de estirpe mesenquimal del tracto genitourinario representan aproximadamente el 5\% de todos los sarcomas y menos del $2 \%$ de todos los tumores urológicos. Los tumores paratesticulares, dentro de los que se incluyen los originados en las túnicas testiculares, epidídimo y cordón espermático, son poco frecuentes y su incidencia es difícil de estimar. En general son neoplasias de gran agresividad con tendencia a la recidiva local y a dar tempranamente metástasis a distancia. Aproximadamente un tercio de los pacientes fallecen por estas causas ${ }^{1,2,23,24}$. 
La información sobre esta entidad y primordialmente de las alternativas terapéuticas adyuvantes tras la cirugía son controversiales ${ }^{23,24}$. Los trabajos de investigación sobre sarcomas paratesticulares son poco frecuentes por su rareza de presentación. Estos se basan en reportes individuales, series de casos y revisiones, cuyas bases de datos no son suficientemente grandes como para analizar la biología de la enfermedad, los factores de riesgo ni llegar a consensos en lo que concierne a su manejo ${ }^{3-6,23,24}$. El Instituto Nacional de Enfermedades Neoplásicas "Eduardo Cáceres Graziani” es el centro de referencia de patología oncológica más importante del país. Tiene la mayor casuística de neoplasias malignas urológicas, dentro de ellas, las de tumores paratesticulares. El presente trabajo identifica y analiza las principales características clínico patologicas, terapéuticas y evolucion de la enfermedad, relacionadas a esta patología.

\section{MATERIAL Y MÉTODOS}

\section{Muestra}

Se revisó retrospectivamente las historias clínicas de los pacientes del departamento de Urología del Instituto Nacional de Enfermedades Neoplásicas desde junio de 1952 hasta diciembre del 2003. Identificadas aquellas con diagnóstico de sarcoma de localización paratesticular, se procedió a recopilar los datos en una ficha recolectora desarrollada para tal fin.

\section{Diagnóstico}

Para corroborar el diagnóstico histopatológico de sarcoma paratesticular, se ubicaron y revisaron con el experto de patología las láminas y tacos de parafina.

\section{Estudio epidemilógico y clínico}

Se obtuvo datos como edad, procedencia, antecedentes patológicos, tiempo de enfermedad, signos, síntomas e información del examen físico.

\section{Estudio imagenológico y laboratorial}

La información radiológica obtenida fue ratificada evaluando el material existente en el archivo del departamento de Radiodiagnóstico (radiografías, tomografías y otros). Los datos de marcadores tumorales como alfa feto proteína y gonadotrofina coriónica humana son de fechas recientes, por lo que sólo se registra en los casos diagnosticados en los últimos años.

\section{Tratamiento}

Se recopiló de las historias clínicas el tipo de tratamiento (quirúrgico, quimioterapia y/o radioterapia), tanto al primario, las metástasis o en caso de recurrencia.

\section{Material Anatomopatológico}

Localización

Los tumores paratesticulares fueron divididos de acuerdo al lado: derecho e izquierdo.

\section{Origen/Ubicación}

Se consideró: cordón espermático, túnicas testiculares, epidídimo y no determinados.

\section{Estudio histológico}

Se revisó el material existente, clasificándolo según la OMS en el capitulo de tumores mesenquimales del escroto, cordón espermático y anexos testiculares (Anexos 1 y 2) ) $^{8}$ También se evaluó el grado de diferenciación, infiltración vascular, perineural, bordes quirúrgicos, compromiso ganglionar y expresión inmunohistoquímica.

Anexo 1. Tumores mesenquimales (WHO-IARC 2004)

\begin{tabular}{ll}
\hline Benignos & Malignos \\
\hline Lipoma & Liposarcoma \\
Leiomioma & Leiomiosarcoma \\
Neurofibroma & Histiocitoma fibroso maligno \\
Tumor de células granulares & Rabdomiosarcoma \\
Angiofibroma celular & \\
Tumor fibroso calcificante & \\
Hamartoma fibroso &
\end{tabular}

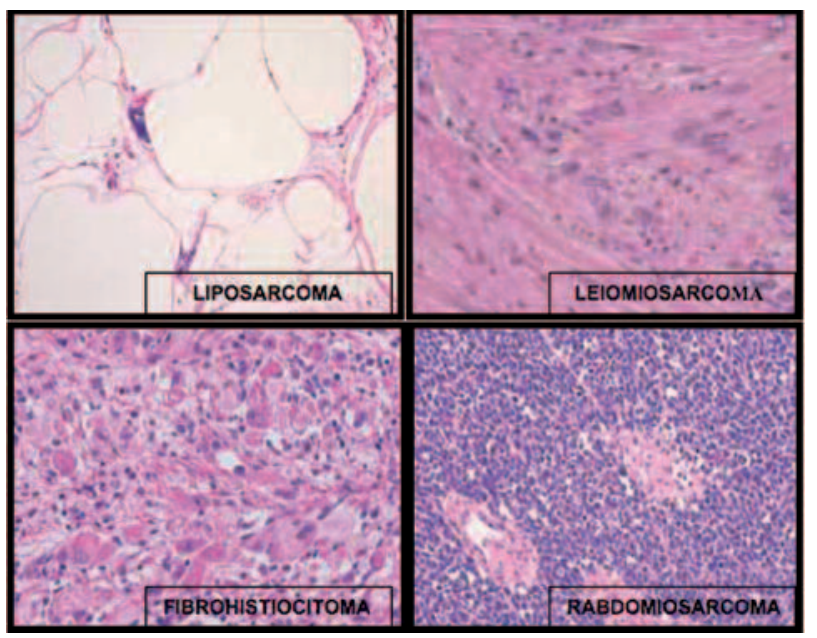

Anexo 2. Tumores mesenquimales malignos (WHO-IARC 2004) 


\section{Estadiaje}

Se utilizó el sistema de estadiaje TNM/ AJCC 2002 basado en el estudio del grado de diferenciación (G), tamaño del tumor (T), compromiso ganglionar $(\mathrm{N})$ y presencia de metástasis (M). En relación al T, todos los casos se consideran profundos ${ }^{1,8,25}$.

\section{Estado de la enfermedad}

$\mathrm{Al}$ revisar las historias clínicas se obtuvo la fecha del último control y el estado de la enfermedad; en los casos sin datos reciente se localizó al paciente o familiares.

\section{Análisis estadístico}

La información fue codificada y procesada utilizando el paquete estadístico SPSS 13,0. Se realizó un análisis descriptivo, los resultados se interpretaron y compararon con los obtenidos en la literatura revisada.

\section{RESULTADOS}

\section{Prevalencia}

Entre junio de 1952 y diciembre del 2003 se diagnosticaron dieciséis (16) casos de sarcoma paratesticular en pacientes adultos del Instituto.

\section{Procedencia y antecedentes}

Seis pacientes $(37,5 \%)$ procedieron de la capital y diez $(6,5 \%)$ de provincias. No se encontró ningún dato de importancia en relación a los antecedentes y/o procedencia.

\section{Edad}

El sarcoma paratesticular en adultos, se presentó con mayor frecuencia en dos picos; uno entre los 16 y 20 años y otro en mayores de sesenta años (Tabla 1).

Tabla 1. Frecuencia de casos de sarcoma paratersticular por edad

\begin{tabular}{lccc}
\hline $\begin{array}{l}\text { Edad } \\
\text { (años) }\end{array}$ & $\begin{array}{c}\text { Tipo } \\
\text { histológico }\end{array}$ & Frecuencia & \% \\
\hline 17 & RMS & 2 & 12,5 \\
18 & RMS & 2 & 12,5 \\
20 & RMS & 3 & 18,8 \\
38 & LMS & 1 & 6,3 \\
45 & RMS & 1 & 6,3 \\
50 & LPS & 1 & 6,3 \\
64 & LPS & 1 & 6,3 \\
65 & NOS & 1 & 6,3 \\
67 & LPS & 1 & 6,3 \\
74 & LMS & 1 & 6,3 \\
88 & LMS & 1 & 6,3 \\
90 & RMS & 1 & 6,3 \\
Total & & $\mathbf{1 6}$ & $\mathbf{1 0 0}$ \\
\hline
\end{tabular}

RMS rabdomiosarcoma; LMS leiomiosarcoma; PLS liposarcoma; NOS sarcoma no especificado.

\section{Cuadro Clínico, Estudios Imageneológicos y Laboratoriales}

La media del tiempo de enfermedad fue 7,5 meses con un rango entre 1 y 24. El 100\% de los pacientes acudieron a consulta al objetivar un tumor de crecimiento progresivo en la región inguíno escrotal, además seis casos tenían sintomatología sistémica en relación a metástasis pulmonar, ósea, retroperitoneal y/o mediastinal.

Al examen clínico, en diez casos (62,5\%), el tumor fue mayor o igual a $10 \mathrm{~cm}$; no hubo predominio de ningún lado distribuyéndose equitativamente (Tabla 2).

Los estudios radiológicos, ecográficos y tomográficos permitieron determinar el estado de la enfermedad al diagnóstico y seguimiento. Los marcadores tumorales, utilizados desde 1997 en el diagnóstico diferencial, en todos los casos fueron negativos.

\section{Tratamiento y estadiaje}

El 100\% fueron sometidos a orquiectomia radical con sección alta del cordón espermático, en tres casos se secciono sobre tumor por estar infiltrado, al estudiarlos encontraron que se trataba de rabdomiosarcomas.

De los nueve casos de rabdomiosarcoma, tres recibieron quimioterapia-radioterapia, dos sólo quimioterapia, uno sólo radioterapia y tres no fueron tributarios de adyuvancia. De los tres casos de leiomiosarcoma dos recurrieron, uno recibió radioterapia local y el otro quimioterapia, el tercero al igual que los otros casos de sarcoma no fue tributario de adyuvancia por razones, como el avanzado estado de la enfermedad o la edad del paciente. En la Tabla 3 se presenta un resumen del tipo de tratamiento recibido, además del estadiaje de acuerdo al TNM/AJCC 2002.

\section{Características anatomopatológicas}

El tipo histológico predominante fue el rabdomiosarcoma (RMS) con nueve casos (56.25\%), seguido del leiomiosarcoma (LMS) y liposarcoma (LPS) con tres casos cada uno $(18,75 \mathrm{c} / \mathrm{u}) \mathrm{y}$ finalmente un caso de sarcoma no especificado (NOS) que pese a la revisión, al ser material muy antiguo no pudo realizarse estudios de Inmunohistoquímica (Fig. 1).

En 13 casos $(81,25 \%)$ el primario estuvo localizado en el cordón espermático, 2 en epidídimo $(12,5 \%)$ y $1(6,25 \%)$ en túnicas testiculares. En tres 
Tabla 2. Características clínicas por paciente y tipo histológico

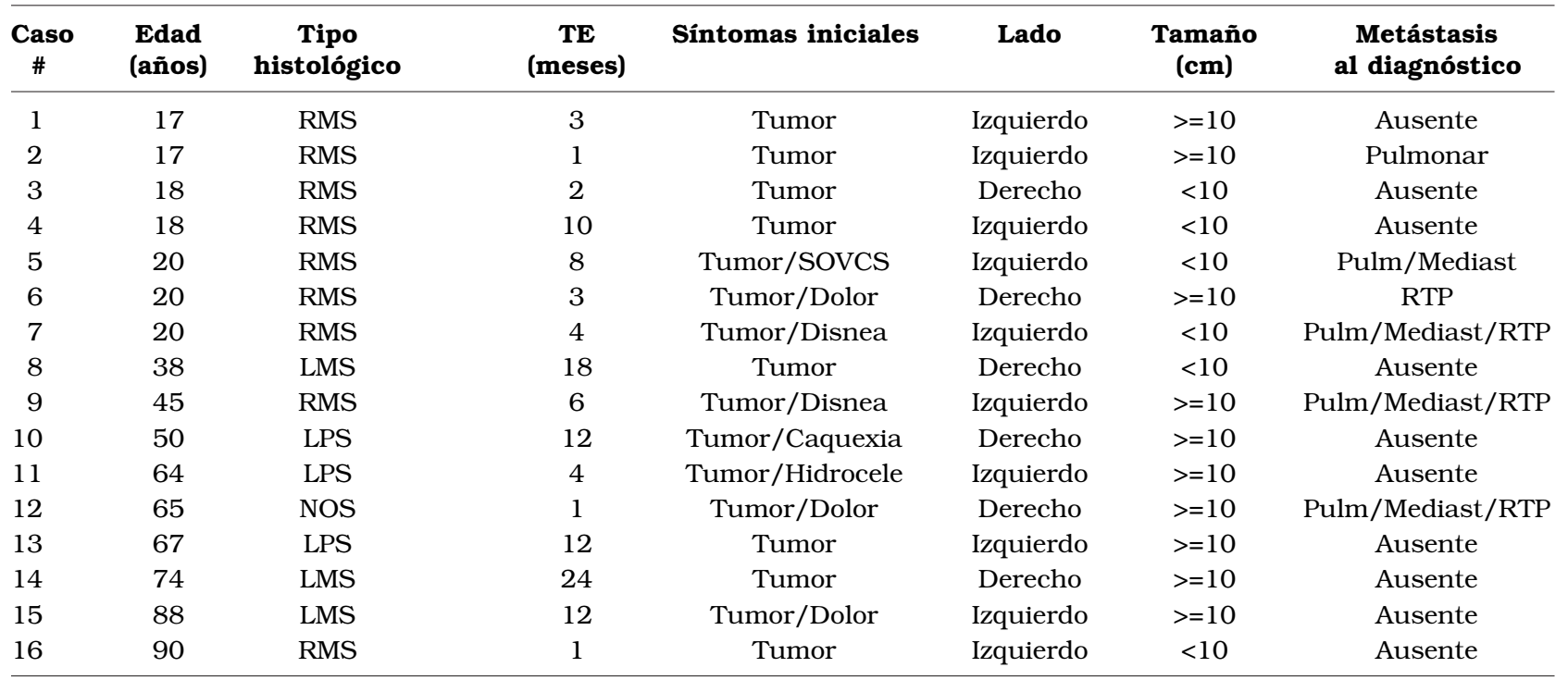

TE: tiempo de enfermedad; RMS: rabdomiosarcoma; LMS: leiomiosarcoma; LPS: liposarcoma; NOS: sarcoma no especificado;SOVCS: sindrome obstructivo vena cava superior; MEDIAST: mediastinal; RTP: retroperitoneal.

Tabla 3. Tratamiento inicial, adyuvante y estadiaje de acuerdo al TNM / AJCC 2002

\begin{tabular}{ccccccccccc}
\hline $\begin{array}{c}\text { Caso } \\
\#\end{array}$ & $\begin{array}{c}\text { Edad } \\
\text { (años) }\end{array}$ & $\begin{array}{c}\text { Tipo } \\
\text { histológico }\end{array}$ & Cirugia & Quimioterapia & Radioterapia & G & T & N & M & Estadio \\
\hline 1 & 17 & RMS & OR & SI & SI & 3 & T2B & 0 & 0 & III \\
2 & 17 & RMS & OR & SI & NO & 3 & T2B & 0 & 1 & IV \\
3 & 18 & RMS & OR & SI & SI & 2 & T1B & 0 & 0 & IA \\
4 & 18 & RMS & OR & NO & SI & 2 & T2B & 0 & 0 & IIA \\
5 & 20 & RMS & OR & SI & NO & 3 & T1B & 0 & 1 & IV \\
6 & 20 & RMS & OR & NO & NO & 3 & T2B & 0 & 1 & IV \\
7 & 20 & RMS & OR & SI & SI & 2 & T1B & 0 & 1 & IV \\
8 & 38 & LMS & OR & SI & NO & 3 & T2B & 0 & 0 & III \\
9 & 45 & RMS & OR & NO & NO & 2 & T2B & 0 & 1 & IV \\
10 & 50 & LPS & OR & NO & NO & 1 & T2B & 0 & 0 & IIA \\
11 & 64 & LPS & OR & NO & NO & 1 & T2B & 0 & 0 & IIA \\
12 & 65 & NOS & OR & NO & NO & 3 & T2B & 0 & 1 & IV \\
13 & 67 & LPS & OR & NO & NO & 2 & T2B & 0 & 0 & IIA \\
14 & 74 & LMS & OR & NO & SI & 2 & T2B & 0 & 0 & IIA \\
15 & 88 & LMS & OR & NO & NO & 2 & T2B & 0 & 0 & IIA \\
16 & 90 & RMS & OR & NO & NO & 2 & T1B & 0 & 0 & IA \\
\hline
\end{tabular}

G: grado de diferenciación; T: tamaño del tumor; N: ganglios; M: metástasis; RMS: rabdomiosarcoma; LMS: leiomiosarcoma; LPS: liposarcoma; NOS: sarcoma no especificado; OR: orquiectomia radical.

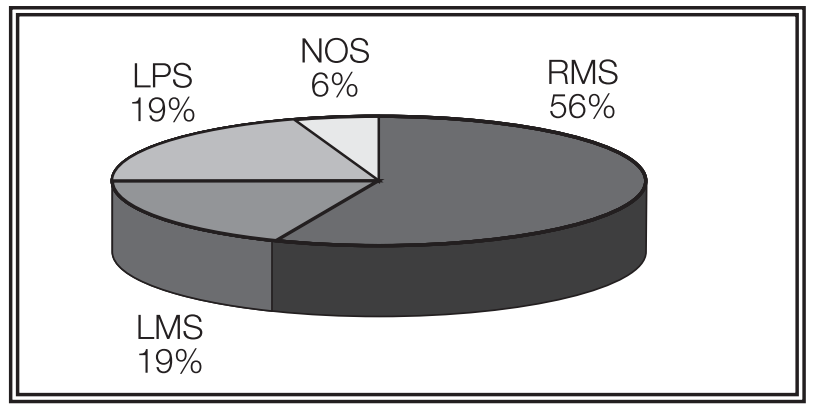

FIGURA 1. Tipos histológicos de sarcomas paratesticulares. de los dieciséis casos (18,75\%), el borde de sección estaba comprometido, dos de ellos infiltraban el testículo y epidídimo; el promedio de tamaño fue de $10.56 \mathrm{~cm}$ con un rango entre 5 y 19 .

En la Tabla 4 se muestran las características histopatológicas de cada caso especificándose el tipo, variedad, tamaño, localización, borde de sección, grado de diferenciación, compromiso del testículo o epidídimo, infiltración vascular, linfática y/o perineural. 
Tabla 4. Características histopatológicas por paciente

\begin{tabular}{|c|c|c|c|c|c|c|c|c|c|c|c|}
\hline $\begin{array}{c}\text { Caso } \\
\#\end{array}$ & $\begin{array}{c}\text { Tipo } \\
\text { histológico }\end{array}$ & Variedad & $\begin{array}{c}\text { Tamaño } \\
\text { (cm) }\end{array}$ & Localización & $\begin{array}{l}\text { Borde } \\
\text { sección }\end{array}$ & $\begin{array}{l}\text { Grado } \\
\text { difer. }\end{array}$ & Epidid. & Test. & IV & IL & IP \\
\hline 1 & RMS & Pleomorfico & 12 & Cordón & Libre & Poco & Libre & Libre & - & - & - \\
\hline 2 & RMS & Embrionario & 11 & Cordón & Comp & Poco & Infilt & Infilt & + & + & + \\
\hline 3 & RMS & Embrionario & 5 & Cordón & Libre & Mod & Libre & Libre & - & - & - \\
\hline 4 & RMS & Pleomorfico & 9 & Cordón & Libre & Mod & Libre & Libre & - & - & - \\
\hline 6 & RMS & Embrionario & 15 & Cordón & Comp & Poco & Infilt & Infilt & + & + & - \\
\hline 7 & RMS & Embrionario & 5 & Cordón & Libre & Mod & Libre & Libre & + & + & - \\
\hline 8 & LMS & Epitelioide & 7 & Cordón & Libre & Poco & Libre & Libre & - & - & - \\
\hline 9 & RMS & Pleomorfico & 13 & Cordón & Comp & Mod & Libre & Libre & + & + & + \\
\hline 10 & LPS & Lipoma like & 11 & Cordón & Libre & Bien & Libre & Libre & - & - & - \\
\hline 11 & LPS & Mixoide & 14 & Cordón & Libre & Bien & Libre & Libre & - & - & - \\
\hline 13 & LPS & Mixoide & 16 & Cordón & Libre & Mod & Libre & Libre & - & - & - \\
\hline 14 & LMS & Convencional & 10 & Cordón & Libre & Mod & Libre & Libre & - & - & - \\
\hline 15 & LMS & Convencional & 19 & Túnica test. & Libre & Mod & Libre & Libre & - & - & - \\
\hline 16 & RMS & Pleomorfico & 5 & Cordón & Libre & Mod & Libre & Libre & - & - & - \\
\hline
\end{tabular}

RMS: rabdomiosarcoma; LMS: leiomiosarcoma; LPS: liposarcoma; NOS: sarcoma no especificado; EPIDID: epidídimo; TEST: testículo; IV: infiltración vascular; IL: infiltración linfática; IP: infiltración perineural.

\section{Estado de la enfermedad y sobrevida}

En la Tabla 5 se presentan el estado de la enfermedad a la fecha de revisión. La sobrevida en meses se considera desde el ingreso hasta el último control o dato obtenido, el promedio fue 32,18 con un rango entre 1 y 142 . Dos pacientes se encuentran vivos, un caso de RMS que recibió quimio-radioterapia, actual- mente sin evidencia de enfermedad y un caso de LMS con recurrencia loco regional, resección local amplia $\mathrm{y}$ en el momento quimioterapia adyuvante.

\section{DISCUSIÓN}

Los tumores malignos de estirpe mesenquimal del tracto genitourinario representan aproximadamente
Tabla 5. Estado de la enfermedad y sobrevida por paciente

\begin{tabular}{ccccccc}
\hline $\begin{array}{c}\text { Caso } \\
\#\end{array}$ & $\begin{array}{c}\text { Tipo } \\
\text { histológico }\end{array}$ & $\begin{array}{c}\text { Estadio } \\
\text { clínico }\end{array}$ & $\begin{array}{c}\text { Fecha } \\
\text { ingreso }\end{array}$ & $\begin{array}{c}\text { Último } \\
\text { control }\end{array}$ & $\begin{array}{c}\text { Estado } \\
\text { enfermedad }\end{array}$ & $\begin{array}{c}\text { Sobrevida } \\
\text { (meses) }\end{array}$ \\
\hline 1 & RMS & III & 09.10 .03 & 11.10 .08 & VSEE & 60 \\
2 & RMS & IV & 18.11 .03 & 15.01 .05 & FCEE & 14 \\
3 & RMS & IA & 23.02 .65 & 25.08 .69 & FCEE & 56 \\
4 & RMS & IIA & 06.11 .74 & 16.12 .76 & FCEE & 25 \\
5 & RMS & IV & 22.08 .01 & 11.10 .01 & FCEE & 2 \\
6 & RMS & IV & 05.11 .02 & 09.12 .02 & FCEE & 1 \\
7 & RMS & IV & 09.07 .03 & 27.12 .04 & FCEE & 17 \\
8 & LMS & III & 27.09 .02 & 11.09 .08 & VCEE & 72 \\
9 & RMS & IV & 21.05 .99 & 15.06 .99 & FCEE & 1 \\
10 & LPS & IIA & 17.02 .97 & 09.11 .98 & FCEE & 21 \\
11 & LPS & IIA & 25.09 .81 & 02.07 .93 & FSEE & 142 \\
12 & NOS & IV & 03.04 .78 & 18.04 .78 & FCEE & 1 \\
13 & LPS & IIA & 03.04 .92 & 31.01 .97 & FSEE & 57 \\
14 & LMS & IIA & 26.02 .03 & 26.04 .04 & FCEE & 14 \\
15 & LMS & IIA & 01.12 .98 & 10.10 .00$. & FCEE & 22 \\
16 & RMS & IA & 22.09 .89 & 11.07 .90 & FSEE & 10 \\
\hline
\end{tabular}

RMS: rabdomiosarcoma; LMS: leiomiosarcoma; LPS: liposarcoma; NOS: sarcoma no especificado; VSEE: vivo sin evidencia de enfermedad; VCEE: vivo con evidencia de enfermedad; FSEE: fallecido sin evidencia de enfermedad; FCEE: fallecido con evidencia de enfermedad. el $5 \%$ de todos los sarcomas y menos del $2 \%$ de todos los tumores urológicos. Los sarcomas de localización paratesticular son una entidad poco frecuente, en nuestra experiencia en el Instituto se diagnosticaron 16 casos entre junio de 1952 y diciembre del 2003. Coincidimos con las no muchas publicaciones en la rareza de esta entidad, de acuerdo a Coleman et al, en una revisión de la literatura se encontró sólo 212 reportes hasta $1978^{2}$.

La mayoría de los pacientes al diagnóstico se presentan con masas escrotales o inguinoescrotales de crecimiento lento, sólidas, irregulares, negativas a la transiluminación y se pueden acompañar de dolor, hidrocele o sintomatología secundaria a las 
metástasis, deben diferenciarse de hernias inguinales, hidroceles, lipomas, hematocele, epididimitis y orquiepididimitis tuberculosa y lesiones malignas como carcinomas y mesoteliomas ${ }^{12}$. Es fundamental en su diagnóstico diferencial el estudio de marcadores tumorales como la alfa feto proteína (AFP) y la gonadotrofina coriónica humana (HCG) $)^{8,12}$. La ecografía es el método diagnóstico más utilizado para su evaluación con una sensibilidad de 95\%. Los estudios de extensión incluyen la radiografía de tórax, tomografía axial computarizada abdóminopélvica y resonancia magnética de la región inguinoescrotal ${ }^{13,14}$. En ésta revisión todos los pacientes acudieron a consulta por presentar un tumor de crecimiento progresivo en la región inguinoescrotal, además seis casos tenían síntomas relacionados a metástasis (disnea, síndrome obstructivo de vena cava superior, dolor y/o caquexia). Los estudios de extensión se realizaron de acuerdo a la época del diagnóstico.

La mayor frecuencia se presento en dos picos, el primero de siete casos entre los 16 y 20 años, todos ellos rabdomiosarcomas, y el segundo de seis casos en mayores de 60 años. En las series publicadas se describen preferentemente en adultos mayores excepto el rabdomiosarcoma que es mas frecuente en las dos primeras décadas de la vida ${ }^{10,11}$.

El estándar de tratamiento quirúrgico inicial es la Orquiectomia radical con sección alta de cordón espermático, siendo para algunos autores suficiente $\mathrm{e}^{23,24,26}$ Sin embargo, aun después de una adecuada cirugía con márgenes negativos, un tercio de los pacientes tienen persistencia de enfermedad microscópica y recurrencia ${ }^{1,2,7}$. En el estudio a 5 años de Ballo et al., se concluye que 19,4\% presentan recurrencia local, 11,1\% metástasis hematógenas y 5,5\% nódulos pelvianos ${ }^{20}$. Otros investigadores consideran el tratamiento adyuvante con quimioterapia y/o radioterapia, las mismas que no han demostrado efectividad con intención curativa. Aproximadamente, un tercio de los pacientes fallecen por enfermedad metastásica ${ }^{7,9,23,24,26}$. En nuestra experiencia todos los pacientes fueron sometidos a orquiectomia radical, en tres casos se seccionó sobre enfermedad por encontrarse el cordón espermático infiltrado, coincidentemente éstos fueron rabdomiosarcomas (RMS) con enfermedad metastásica en pulmón, mediastino y/o retroperitoneo. De los casos con diagnóstico anatomopatológico de RMS, tres recibieron adyuvancia con quimio-radioterapia, dos solo quimioterapia, uno solo radiotera- pia y tres por el estado de la enfermedad o la edad del paciente no fueron tributarios de otro tratamiento. Dos casos de leiomiosarcoma recurrieron, uno recibió radioterapia sin respuesta y otro fue sometido a resección local amplia y quimioterapia.

El principal patrón de diseminación de los sarcomas del cordón es por contigüidad a través del canal inguinal y de éste a la cavidad abdominal, menos frecuentes son las vía hematógena y/o linfática, ésto explica porque hasta la fecha la linfadenectomia retroperitoneal no ha demostrado cambios en el pronóstico ni sobrevida ${ }^{2,20,21}$. El rol de la disección ganglionar retroperitoneal permanece controversial, ha sido recomendada por algunos autores en pacientes con rabdomiosarcoma y fibrohistiocitoma maligno de alto grado, en el caso de los leiomiosarcomas y liposarcomas su uso no está aceptado ${ }^{1,22}$.

La quimioterapia complementaria no está bien establecida, solo es aceptada en niños con rabdomiosarcoma en quienes tiene un efecto significativo en la sobrevida, en adultos los resultados no son convincentes $^{1,2}$. Un meta-análisis de 14 ensayos randomizados de varios centros oncológicos mostraron que la quimioterapia con doxorrubicina prolonga el tiempo de recurrencia local y de sobrevida, pero no está estadísticamente comprobado ${ }^{1,21}$.

El comportamiento biológico del tumor es variable de acuerdo al tipo histológico, hay que considerar que la distinción entre las variedades de sarcomas es aun más difícil por lo que se utilizan técnicas especiales como la inmunohistoquímica ${ }^{15}$.

Los liposarcomas pueden desarrollarse en cualquier sitio donde exista tejido adiposo, su localización principal es el retroperitoneo y representan el 20\% de los sarcomas paratesticulares, siendo la mayor serie publicada de 30 casos $^{3,8}$. Se dividen en 5 subtipos: bien diferenciados, mixoide/células redondas, lipoblástico, fibroblástico y pleomórfico. La mayoría son bien diferenciados (40-45\%), con sus variantes esclerosante, "lipoma-like" e inflamatorio; en el 5 a $10 \%$ puede observarse combinación de las mismas ${ }^{1,3,8}$. Nosotros encontramos tres casos, dos mixoides y uno bien diferenciado variante "lipoma like" el cual recurrió a distancia en pulmón a los 19 meses.

Los leiomiosarcomas en adultos representan el $30 \%$ de los sarcomas paratesticulares. Se presentan como una masa nodular, mayormente cerca del cordón espermático y menos frecuente en el epidídimo, completamente separado del testículo. El pico de incidencia está en la sexta y séptima década de la vida. Su comportamiento y pronóstico es variable. 
Histológicamente tiene patrones bien diferenciados hasta poco diferenciados con rasgos anaplásicos, que requiere del estudio inmunohistoquimico con actina músculo liso para su confirmación ${ }^{1,5,8,18}$. En nuestro trabajo se presentaron tres casos, uno de ellos recurrió localmente.

El rabdomiosarcoma paratesticular es un tumor raro, resulta excepcional en el adulto y se presenta sobre todo en las dos primeras décadas de la vida. A pesar de esto representa el $24 \%$ de los tumores paratesticulares del adulto, $80 \%$ de los cuales ocurren antes de los 21 años ${ }^{24}$. Su histogénesis esta relacionada con el desarrollo embrionario del cordón, epidídimo, cubiertas testiculares y tejido conectivo. Aunque macroscópicamente parecen ser bien circunscritos, el examen microscópico revela un patrón infiltrativo. Se describen tres tipos histológicos: pleomórfico, alveolar y embrionario, siendo éste último el más frecuente ${ }^{4,8,19}$. En esta serie, siete de nueve casos se presentaron en los últimos años de la segunda década y otros dos en pacientes de 45 y 90 años, los tipos histológico presentados fueron embrionario en cinco casos (55,5\%) y pleomórfico en cuatro (44,5\%).

El fibrohistiocitoma maligno pleomórfico es un diagnóstico histológico hecho por exclusión de las otras entidades con estudio inmunohistoquímico. Se reconocen cuatro subtipos: mixoide, inflamatorio, de células gigantes y estoriforme-pleomórfico; éste último es el más frecuente en la localización paratesticular. Tiene como característica peculiar la recurrencia, que alcanza hasta el 51\% en algunos trabajos ${ }^{8,24}$.

Un caso diagnosticado en 1978 no pudo ser clasificado ya que el material de patología por el estado de conservación no pudo ser sometido a estudios inmunohistoquímicos.

Catorce pacientes fallecieron, once $(68,7 \%)$ por progresión de enfermedad y tres $(18,75 \%)$ sin evidencia de enfermedad, de éstos, dos casos eran portadores de liposarcoma con sobrevidas de 57 y 142 meses y uno de rabdomiosarcoma, pero tenía 90 años. Sólo dos pacientes se encuentran vivos, uno con rabdomiosarcoma que recibió quimioterapia y radioterapia, actualmente sin evidencia de enfermedad y otro con leiomiosarcoma con recurrencia loco regional por lo que fue sometido a resección local amplia y en el momento recibe quimioterapia adyuvante.

Definitivamente aun no se ha dicho todo sobre el comportamiento y manejo de esta entidad y sus variantes, queda mucho por investigar en la búsqueda de mejorar la sobrevida libre de enfermedad y porque no, encontrar la terapia con intención curativa.

\section{REFERENCIAS}

1. Khoubehi B, Mishra V, Ali M, Motiwala H and Karim O. Adult paratesticular tumours. BJU International. 2002;90(7):707-715

2. Coleman J, Brennan M, Alektiar K, Russo P. Adult Spermatic Cord Sarcomas: Management and results. Ann Surg Oncol. 2003;10(6):669-675.

3. Peyrí Rey E, Urban Ramón A, Martínez Fernández M, Sanmarti Da Silva B. Liposarcoma dediferenciado del cordón espermático: Degeneración de un lipoma previo resecado. Actas Urol Esp. 2003;27(5):383-386.

4. Astigueta Pérez A, Pow-Sang Godoy M, Abad Licham M, Sánchez Lihon J, Morante Deza C, Meza Montoya L, et al. Sarcoma mixto paratesticular: Liposarcoma - Rabdomiosarcoma. Presentación de un caso y revisión de la literatura. Arch Esp Urol. 2007;60(10):1204 -1208

5. Eslami F, Verghese M and Shmookler B. Paratesticular Leiomyosarcoma in an elderly man. Urology 2000; 60: 1112-2002. Evans H. Liposarcomas and atypical lipomatous tumors: a study of 66 cases followed for a minimum of 10 years. Surg Pathol 1988; 1: 41-54.

6. Evans H. Liposarcomas and atypical lipomatous tumors: a study of 66 cases followed for a minimum of 10 years. Surg Pathol 1988;1:41-54.

7. Dotan ZA, Tal R, Golijanin D, Snyder ME, Antonescu C, Brennan MF, et al. Adult genitourinary sarcoma: the 25 year Memorial Sloan Kettering experience. J Urol 2006;176(5):2033-2038.

8. Eble J, Sauter G, Epstein J and Sesterhenn I. Tumours of the testis and paratesticular tissue. En Tumours of the urinary system and male genital organs. Lyon France. WHO IARC press; 2004; 267-273.

9. Mondaini N, Palli D, Saieva C, Nesi G, Franchi A, Ponchietti R, et al. Clinical characteristics and overall survival in genitourinary sarcomas treated with curative intent: a multicenter study. Eur Urol. 2005;47(4):468-473.

10. Cao E, Alarcón H, Fuster J, Lopez P, Linares E y Perez M. Tumores testiculares y paratesticulares prepuberales. Experiencia en nuestro centro y revisión de la literatura. Actas Urol Esp 2005; 29 (4): 355359.

11. Piyush K. Agarwal and Jeffrey S. Testicular and paratesticular neoplasms in prepuberal males. J Urol 2006; 176: 875-881. Folpe A and Weiss S. Paratesticular soft tissue neoplasms. Semin Diag Pathol 2000; 17: 307-318.

12. Folpe A and Weiss S. Paratesticular soft tissue neoplasms. Semin Diag Pathol 2000; $17: 307-318$

13. Akbar S, Sayyed T, Jafri S, Hasteh F, Nelly J. Multimodality imagin of paratesticular neoplasms and their rare mimics. Radiographics. 2003;23(6):1461-1476.

14. Benson C, Doubilet P, Richie J. Sonography of the male genital tract. AJR. 1989;153(4):705-713.

15. Dabbs David. Inmunohistology of the prostate, bladder, testis and kidney. En Diagnostic inmunohistochemestry. Philadelphia. Churchill Livingstone Elsevier 2006. 509-610.

16. Suster S, Wong TY, Moran CA. Sarcomas with combined features of liposarcomas and leiomyosarcoma. Study of two cases of an unusually soft tissue tumor showing dual lineage differentiation. Am J Surg Pathol. 1993;17(9):905-911.

17. Folpe AL, Weiss SW. Lipoleiomyosarcoma (well-differentiated liposarcoma with leiomyosarcomatous differentiation): a clinicopathologic study of nine cases including one with dedifferentiation. Am J Surg Pathol. Jun. 2002;26(6):742-749.

18. Forrest $\mathrm{M}$ and Ahlering T. Bilateral asynchronous paratesticular leiomiosarcomas. J Urol. 2001;166(6):2309-2310.

19. Mora N, Ponce C, Llopis M et al. Rabdomiosarcoma Paratesticular. Actas Urol Esp. 2004;28(2):245-248.

20. Ballo MT, Zagars GK, Pisters PW, Feig BW, Patel SR, von Eschenbach AC Spermatic Cord Sarcoma: Outcome, Patterns of failure and management. J Urol 2001;166(4):1306-1310.

21. Catton C, Jewett M, O'sullivan B, Kandel R. Paratesticular Sarcoma: Failure patterns after definitive local therapy. J Urol. 1999;161(6):1844-1847.

22. Hermans BP, Foster RS, Bihrle R, Little S, Sandler A, Einhorn LH, et al. Is retroperitoneal lymph node dissection necessary for adult paratesticular rhabdomyosarcoma?. J Urol. 1998; 160: 2074-2077.

23. Walsh P, Retik A, Vaughan E, Wein A. Neoplasias del testiculo y anexos. En Campbell Urología. Saunders Philadelphia. Elsevier Science. 2002. 3187-3191.

24. Resel L, Moreno J. Tumores paratesticulares. En: Tratado de Urología Oncológica. Grupo Saned 2003. 1037-1045.

25. Greene F, Page D, Fleming I, Fritz A, Balch C, Haller D et al. eds. American Joint Committee on Cancer. AJCC Cancer staging manual, sixth edition. SpringerVerlag New Yorc Inc. 2002. 193-197.

26. Vogelzang N, Scardino P, Shipley W, Coffey D. Genitourinary sarcomas and carcinosarcomas in adults. En: Comprehensive textbook of Genitourinary Oncology Lippincott Williams \& Wilkins 2000:1102-1119.

Correspondencia autor: Dr. Juan C. Astigueta Pérez

Departamento de Cirugía Urológica. Instituto Regional de

Enfermedades Neoplásicas - Norte

Panamericana Norte km 558, Trujillo, Perú

E-mail autor: jca astigueta@hotmail.com

Información artículo: Original - Cáncer de testículo

Trabajo recibido: noviembre 2008

Trabajo aceptado: diciembre 2008 